\title{
ERRATUM
}

Takehiro Okuda · Takashi Noda · Tomoko Yamamoto

Norihiko Ito · Masahiro Nakaoka

\section{Latitudinal gradient of species diversity: multi-scale variability in rocky intertidal sessile assemblages along the Northwestern Pacific coast}

Published online: 31 August 2004

(C) The Society of Population Ecology and Springer-Verlag Tokyo 2004

\section{Popul Ecol (2004) 46:159-170}

tion of the Simpson's diversity index column. The correct table appears here.

An error appeared in Table 4: the figures 0.000 and 0.857 were transposed in the Variable-level-model sec-

Table 4 Results of the hierarchical linear models applied to examine whether latitude affects the slope or intercept of the regression equations for $\bar{\alpha}_{2}$ against $\alpha_{3}$. In the analyses, both latitude and $\alpha_{3}$ were treated as fixed independent variables

\begin{tabular}{|c|c|c|c|c|}
\hline \multirow[t]{2}{*}{ Independent variables } & \multicolumn{2}{|l|}{ Species richness } & \multicolumn{2}{|l|}{ Simpson's diversity index } \\
\hline & $\begin{array}{l}\text { Estimated regression coefficient } \\
(\text { mean } \pm \text { SE) }\end{array}$ & $P$ & $\begin{array}{l}\text { Estimated regression coefficient } \\
(\text { mean } \pm \mathrm{SE})\end{array}$ & $P$ \\
\hline \multicolumn{5}{|l|}{ Variable-slope-model } \\
\hline Intercept & $-10.236 \pm 6.425$ & 0.111 & $-0.512 \pm 0.500$ & 0.305 \\
\hline Latitude & $0.354 \pm 0.165$ & 0.032 & $0.014 \pm 0.013$ & 0.277 \\
\hline$\alpha_{3}$ & $0.469 \pm 0.262$ & 0.073 & $1.533 \pm 0.673$ & 0.023 \\
\hline Latitude $\times \alpha_{3}$ & $\begin{array}{l}-0.003 \pm 0.007 \\
\text { Log likelihood: }-48.841 \text { AIC: } 22.54\end{array}$ & 0.646 & $\begin{array}{l}-0.019 \pm 0.017 \\
\text { Log likelihood: }-192.691 \text { AIC: }-44.58\end{array}$ & 0.284 \\
\hline \multicolumn{5}{|l|}{ Variable-level-model } \\
\hline Intercept & $-7.544 \pm 2.655$ & 0.004 & $0.015 \pm 0.092$ & 0.874 \\
\hline Latitude & $0.283 \pm 0.058$ & 0.000 & $0.000 \pm 0.002$ & 0.857 \\
\hline$\alpha_{3}$-diversity & $0.350 \pm 0.034$ & 0.000 & $0.815 \pm 0.065$ & 0.000 \\
\hline \multicolumn{5}{|c|}{ o the } \\
\hline Intercept & $4.908 \pm 0.974$ & 0.000 & $0.029 \pm 0.046$ & 0.532 \\
\hline$\alpha_{3}$-diversity & $0.268 \pm 0.039$ & 0.000 & $0.813 \pm 0.064$ & 0.000 \\
\hline & Log likelihood: -57.704 AIC: 36.27 & & Log likelihood: -192.717 AIC: -48.15 & \\
\hline
\end{tabular}

The online version of the original article can be found at http:// dx.doi.org/10.1007/s10144-004-0185-9

T. Okuda $\cdot$ T. Noda $(\bowtie)$

Graduate School of Fisheries Sciences,

Hokkaido University, 3-1-1 Minato-cho,

Hakodate 041-8611, Japan

E-mail: noda@fish.hokudai.ac.jp

Tel.: + 81-138-405549

Fax: + 81-138-405547

T. Yamamoto

Faculty of Fisheries Sciences, Kagoshima University,

Kagoshima, Japan

N. Ito $\cdot$ M. Nakaoka

Graduate School of Science and Technology,

Chiba University, Inage, Japan 